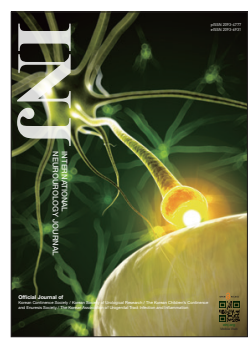

\title{
Reply to Commentary on "Repetitive Transcranial Magnetic Stimulation for Chronic Prostatitis/Chronic Pelvic Pain Syndrome: A Prospective Pilot Study"
}

\author{
Jussi Nikkola ${ }^{1,2}$, Anu Holm ${ }^{3,4}$, Marjo Seppänen ${ }^{1}$, Teemu Joutsi ${ }^{1}$, Esa Rauhala ${ }^{3}$, Antti Kaipia ${ }^{2}$ \\ ${ }^{1}$ Department of Urology, Tampere University Hospital, Tampere, Finland \\ ${ }^{2}$ Department of Surgery, Satakunta Hospital District, Pori, Finland \\ ${ }^{3}$ Unit of Clinical Neurophysiology, Satakunta Hospital District, Pori, Finland \\ ${ }^{4}$ Faculty of Health and Welfare, Satakunta University of Applied Sciences, Pori, Finland
}

We gladly welcome the discussion in the commentary [1]. Chronic prostatitis/chronic pelvic pain syndrome (CP/CPPS) patients indeed lack novel treatment options and unfortunately research interest on treatment-resistant pain associated with long-lasting CP/CPPS has been scarce. Since CP/CPPS would be a new indication for repetitive transcranial magnetic stimulation (rTMS), the aim of this feasibility study was to assess if rTMS would have any effect on pain in CP/CPPS in order to give support for future randomized controlled trials.

As discussed in the article [2] and in the commentary, the findings fit the typical response profile of rTMS. In clinical use on depression and pain indications, our institutional protocol is modified from study by Hodaj et al. [3]. It consists of intensive period of 10 sessions during 2 weeks followed by 8 maintenance sessions over the first 2 months: 2 weekly sessions for the first 2 weeks, 1 weekly session for the next 2 weeks and 1 session in 2 weeks then after. After that, the interval of maintenance treatment can be lengthened. In pain indications 1-2 sessions per month is typical in patients receiving positive response and undergoing maintenance treatment.

The current recommendations for rTMS state, that there still is not unequivocal maintenance treatment algorithm available [4]. The present study encourages future research on rTMS for $\mathrm{CP} / \mathrm{CPPS}$ and show that at least moderate treatment response can be reached even with short treatment and without mainte- nance. We encourage future randomized controlled trials to study also the role of maintenance sessions.

- Conflict of Interest: No potential conflict of interest relevant to this article was reported.

\section{REFERENCES}

1. Shin YS, Lee KS, Kam SC. Commentary on "Repetitive transcranial magnetic stimulation for chronic prostatitis/chronic pelvic pain syndrome: a prospective pilot study". Int Neurourol J 2020;24:296.

2. Nikkola J, Holm A, Seppänen M, Joutsi T, Rauhala E, Kaipia A. Repetitive transcranial magnetic stimulation for chronic prostatitis/ chronic pelvic pain syndrome: a prospective pilot study. Int Neurourol J 2020;24:144-9.

3. Hodaj H, Alibeu JP, Payen JF, Lefaucheur JP. Treatment of chronic facial pain including cluster headache by repetitive transcranial magnetic stimulation of the motor cortex with maintenance sessions: a naturalistic study. Brain Stimul 2015;8:801-7.

4. Lefaucheur JP, Aleman A, Baeken C, Benninger DH, Brunelin J, Di Lazzaro V, et al. Evidence-based guidelines on the therapeutic use of repetitive transcranial magnetic stimulation (rTMS): an update (2014-2018). Clin Neurophysiol 2020;131:474-528.
Corresponding author: Jussi Nikkola (iD https://orcid.org/0000-0002-5077-2012 Department of Urology, Tampere University Hospital, Kuntokatu 2, 33520 Tampere, Finland

E-mail: jussi.nikkola@fimnet.fi

Submitted: July 28, 2020 / Accepted after revision: August 23, 2020
(7) $\$$ This is an Open Access article distributed under the terms of the Creative Commons Attribution Non-Commercial License (https://creativecommons.org/licenses/by-nc/4.0/) which permits unrestricted non-commercial use, distribution, and reproduction in any medium, provided the original work is properly cited. 